\title{
0036. Confocal imaging of impaired mitochondrial function in the cerebral cortex of rats during haemorrhagic shock in vivo
}

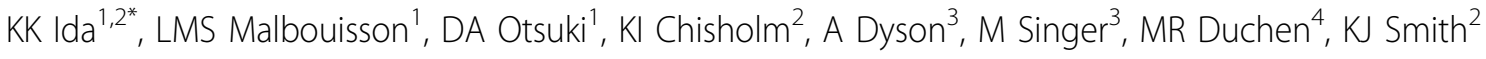 \\ From ESICM LIVES 2014 \\ Barcelona, Spain. 27 September - 1 October 2014
}

\section{Introduction}

Haemorrhagic shock (HS)-induced hypotension impairs cerebral perfusion pressure and decreases oxygen supply to the brain (1), but the consequences for brain mitochondrial function are poorly understood.

\section{Objectives}

To investigate the effects of HS on mitochondrial function in the cerebral cortex of rats in vivo.

\section{Methods}

A flap of skull was removed and cerebral cortex exposed in isoflurane-anaesthetised rats undergoing $\mathrm{HS}$ (target mean arterial pressure (MAP) of $40 \mathrm{mmHg}$ for 30 minutes) $(n=7)$ compared with sham $(n=6)$ controls. Mitochondrial function was assessed by observing mitochondrial membrane potential (using tetramethyl rhodamine methyl ester (TMRM); ex: $543 \mathrm{~nm}$; em: $585 \mathrm{~nm}$ ), and endogenous flavin adenine dinucleotide (FAD) autofluorescence (ex: $488 \mathrm{~nm}$; em: 505-570 nm) by confocal imaging. The TMRM and FAD signals were registered before induction of HS (baseline), at MAP of $40 \mathrm{mmHg}$ (shock), and at 30 (T30) and 60 (T60) minutes after shock.

\section{Results}

There was a loss of mitochondrial membrane potential (decreased TMRM signal; $p<0.001$ ) and reduction of the $\mathrm{FAD} / \mathrm{FADH}_{2}$ pair (seen as decreased FAD fluorescence; $p<0.01$ ) at both T30 and T60 post-shock, except for a striking protection observed as a 'halo' extending $\sim 30-40 \mathrm{~mm}$ from arterioles (Figure 1). No loss of mitochondrial function was observed in sham controls (MAP >90 mmHg in all animals at both T30 and T60).

\section{Conclusions}

HS-induced hypotension causes a marked loss of mitochondrial membrane potential and FAD fluorescence in cerebral cortex, except in regions in immediate proximity to arterioles. This study reveals a profound spatial vulnerability of cortical tissue to reduced blood flow.

Grant acknowledgment

Programa Ciência sem Fronteiras 10464-4-12, UK MS Society, MRC.

\section{Authors' details}

${ }^{1}$ University of São Paulo, Medical School, Anaesthesiology LIM-8, São Paulo, Brazil. ' University College London, Department of Neuroinflammation,

Institute of Neurology, London, UK. ${ }^{3}$ University College London, Bloomsbury Institute of Intensive Care Medicine, London, UK. ${ }^{4}$ University College London, Department of Cell and Developmental Biology, London, UK.

\section{Published: 26 September 2014}

\section{Reference}

1. Cavus E, Meybohm P, Doerges V, et al: Cerebral effects of three resuscitation protocols in uncontrolled haemorrhagic shock: a randomised controlled experimental study. Resuscitation 2009, 80:567-72.

doi:10.1186/2197-425X-2-S1-09

Cite this article as: Ida et al:: 0036 . Confocal imaging of impaired mitochondrial function in the cerebral cortex of rats during haemorrhagic shock in vivo. Intensive Care Medicine Experimental 2014 2(Suppl 1):09. 
A Haemorrhagic shock
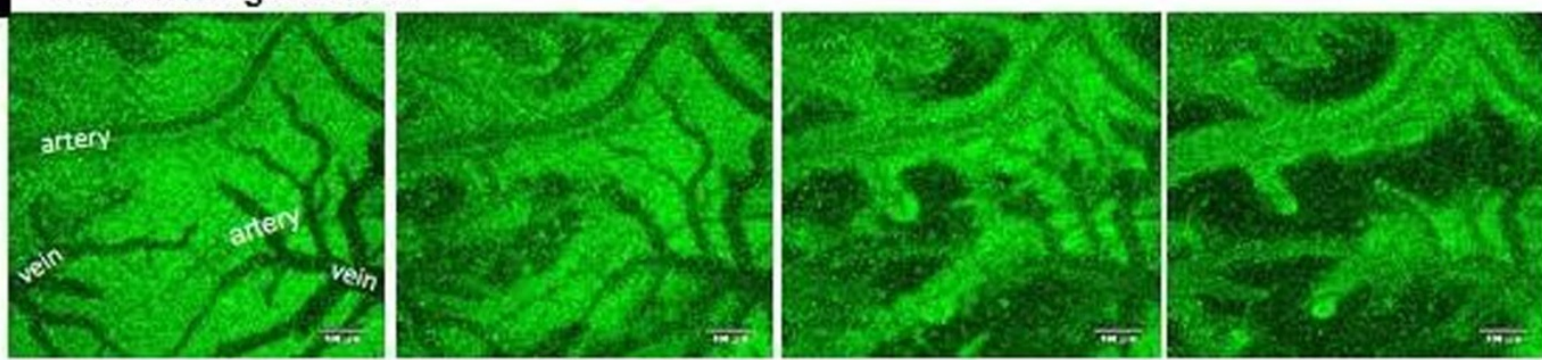

Sham

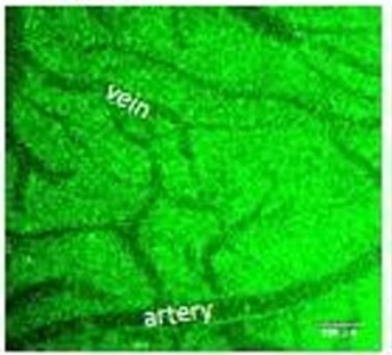

Baseline

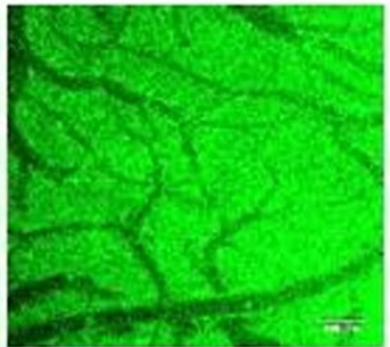

Shock

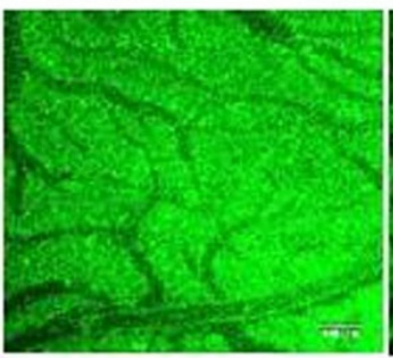

30 minutes after shock

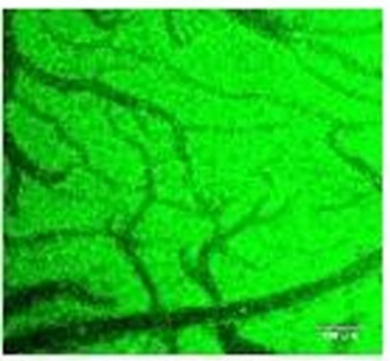

60 minutes after shock

B

\section{Ratio of fluorescence intensity of the halo Arteriolar:Venular}

I. FAD

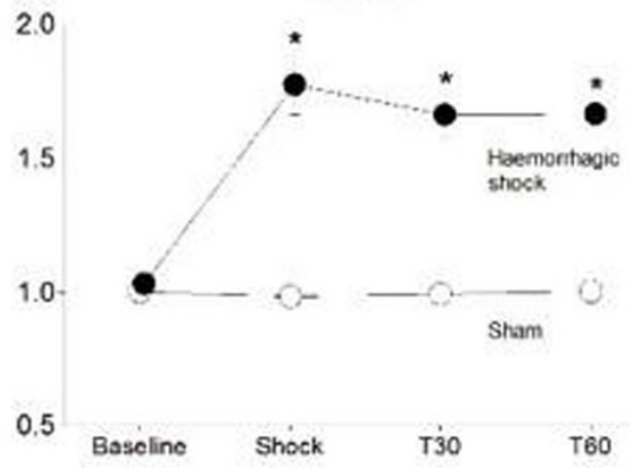

II. TMRM at T60

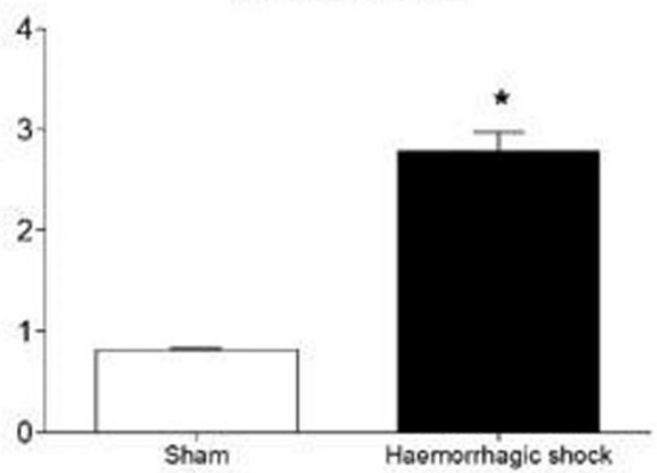

Figure 1 (A) Images of the somatosensory cortex at baseline, haemorrhagic shock, and at 30 and 60 minutes after shock. Green mitochondrial FAD autofluorescence indicates an oxygenated environment, and in shick this is only maintained within $\sim 30-40 \mu \mathrm{m}$ of arteries. (B) Ratio of the FAD (I) and TMRM (II) fluorescence intensity of the halo extending 30-40 $\mu \mathrm{m}$, from arterioles/arteries to venules/veins in rats undergoing haemorrhagic shock. *Within a time-point, the value is significantly different from sham $(p<0.01)$. 